\title{
STUDIES OF THE ISM IN THE LMC USING SN1987A
}

\author{
MAX PETTINI \\ Anglo-Australian Observatory \\ PO Box 296 \\ Epping NSW 2121 \\ Australia
}

\begin{abstract}
The exceptional brightness of SN1987A provided a wealth of opportunities for probing not only the interstellar medium in our Galaxy and in the Large Magellanic Cloud (LMC), but also any intergalactic matter between the two. Spectroscopic work has been directed both towards searches for very weak absorption lines, which require data of exceptionally high signal-to-noise ratio, and towards recording spectra of known features at unprecedentedly high resolution. Both approaches have yielded exciting and unexpected results. The first detection of $[\mathrm{FeX}]$ absorption has revealed the presence of million-degree gas in the interstellar medium of the LMC, possibly resulting from the explosions of previous supernovae in the 30-Doradus HII region. The ultra-high-resolution observations have been successful in resolving the hyperfine structure of the sodium D lines in several interstellar clouds along the line of sight to the supernova. This implies that the clouds are at temperatures of, at most, $170 \mathrm{~K}$ and have internal turbulent velocities of not more than $0.2 \mathrm{~km} \mathrm{~s}^{-1}$; large-scale motions thus appear to be mainly subsonic in these clouds. Radio observations of $\mathrm{HI}$ emission at $21-\mathrm{cm}$ with the Parkes telescope have been combined with measurements of a variety of ultraviolet absorption lines, obtained with the International Ultraviolet Explorer satellite, to give the most detailed picture yet of the chemical composition of the gas between the Galaxy and the LMC. Finally, photographic monitoring of the light echo of SN 1987A over the last two years has provided a three-dimensional view of the interstellar environment in which SN 1987A exploded, complementing vividly the information deduced from the spectroscopic results.
\end{abstract}

